\title{
Resolving and Mediating Ambiguous Contexts for Pervasive Care Environments
}

\author{
${ }^{1}$ Nirmalya Roy, ${ }^{1}$ Christine Julien and ${ }^{2}$ Sajal K. Das \\ ${ }^{1}$ The Department of Electrical and Computer Engineering \\ The University of Texas at Austin \\ Email: \{nirmalya.roy, c.julien\}@mail.utexas.edu \\ ${ }^{2}$ The Department of Computer Science and Engineering \\ The University of Texas at Arlington \\ Email: das@uta.edu
}

\begin{abstract}
Ubiquitous (or smart) healthcare applications envision sensor rich computing and networking environments that can capture various types of contexts of patients (or inhabitants of the environment), such as their location, activities and vital signs. Such context information is useful in providing health related and wellness management services in an intelligent way so as to promote independent living. However, in reality, both sensed and interpreted contexts may often be ambiguous, leading to fatal decisions if not properly handled. Thus, a significant challenge facing the development of realistic and deployable context-aware services for healthcare applications is the ability to deal with ambiguous contexts to prevent hazardous situations. In this work, we propose a quality assured context mediation framework, based on efficient context-aware data fusion and information theoretic system parameter selection for optimal state estimation in resource constrained sensor network. The proposed framework provides a systematic approach based on dynamic Bayesian network to derive context fragments and deal with context ambiguity or error in a probabilistic manner. It has the ability to incorporate context representation according to the applications' quality requirement. Experimental results using SunSPOT sensors demonstrate the promise of this approach.
\end{abstract}

Keywords: Context-awareness, Ambiguous contexts, Bayesian networks, Multi sensor fusion, Information theory.

\section{OUR CONTRIBUTIONS}

Our approach fuses data from disparate sensors, represents abstract context state, and reasons efficiently about this state, to support context-aware services that handle ambiguity. Our goal is to build a framework that resolves information redundancy and also ensures the conformance to the application's quality of context (QoC) bound based on an optimal sensor configuration. We state an optimization problem using a generic QoC function to determine the optimal tolerance range of the sensors that satisfy the specified quality of context at a minimum communication cost. Then we propose a Dynamic Bayesian Networks (DBNs) based model that uses the sensed data to interpret context state through fusion and an information theoretic reasoning technique to select the optimal sensor data values to minimize ambiguity. We build a system using various SunSPOT sensors for sensing and mediating user context state. Experiments demonstrate that the proposed framework is capable of determining the user context state and reducing the sensing overhead while ensuring acceptable context accuracy.

This paper is organized as follows. Section II describes the basic concepts of our context model and the quality of context (QoC) optimization problem. Section III describes the context-aware data fusion model based on DBNs for resolving ambiguity. In Section IV we study the structure of an optimal sensor configuration to minimize the state estimation error from an information theoretic point of view.

\section{Context Model}

Context-aware data fusion in the face of ambiguities is challenging because the data in sensor networks is inherently uncertain. We make use of a space-based context model [4] and extend it with quality of context (QoC) attributes. This model captures the underlying description of context related knowledge such as context attribute $\left(a_{i}\right)$, context state $\left(S_{i}\right)$ and situation space $\left(\mathcal{R}_{i}\right)$, and attempts to incorporate various intuitions that should impact context inference to produce better fusion results. For specific definitions of these parameters see [5].

\section{A. Quality of Context Model}

Despite recent developments in sensing and network technology, continuous monitoring of context is still challenging due to resource constraints. Consequently, the amount of information transmitted to a fusion mediator should be minimized to prolong network lifetime. The idea of exploiting temporal correlation across successive samples of individual sensors to reduce communication overhead is addressed in [2]. The focus there was on meeting the quality requirements for a particular class of aggregation queries, whereas we focus on arbitrary relationships between a context state and the underlying sensor data. Thus we define Quality of Context (QoC) [3] as a metric for minimizing resource usage. We assume that the application processes an aggregation query with its QoC specified by a precision range $Q$, which implies that the aggregate value computed at the mediator at any instant should be accurate within $\pm Q$.

\section{CONTEXT-AwARE DATA FUSION}

A characteristic of a sensor-rich smart healthcare environment is that it senses and reacts to context, information sensed 
about the environment's occupants and their daily activities, by providing context-aware services that facilitates the occupants in their everyday actions. Here we develop an approach for sensor data fusion in context-aware healthcare environment considering the underlying space-based context model and a set of intuitions it covers. In the case of context-aware services, it is really difficult to get an accurate and well defined context which we can classify as 'unambiguous' since the interpretation of sensed data as context is mostly imperfect and ambiguous. To alleviate this problem, we propose a DBN model. Thus, we design a context-aware (active) data fusion framework based on DBNs to reduce this ambiguity as much as possible during the situation inference (patient's behavior or sickness) process.

\section{INFORMATION THEORETIC REASONING}

Considering that most sensors are battery operated and use wireless communication, energy-efficiency is important in addition to managing changing QoC requirements. For example, higher quality might be required for certain healthrelated context attributes during high stress situations such as a medical emergency, and lower quality during low stress situations such as sleep. Fig. 1 shows the context attributes requirement graph for a personal health monitor and includes multiple states for each vital signs that can be monitored depending upon the context state of the patient. For example, the Fig. 1 shows that when a patient is lying in a distressed state and the blood pressure is low, the blood oxygen level must be monitored with a quality of .7 and the blood pressure must be monitored with a quality of .8 . So the problem here is to decide what type of information each sensor should send to the fusion center to estimate the best current state of the patient while satisfying the application QoC requirements for each context attribute by minimizing the state estimation error.

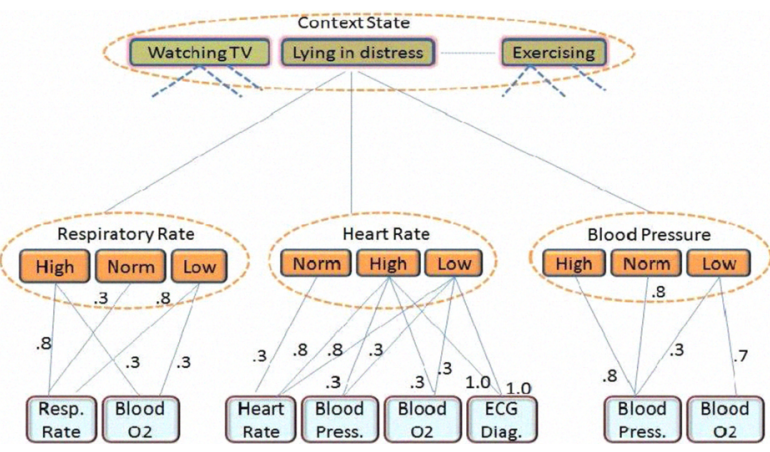

Fig. 1. State-based Context attribute requirement graph with the required QoC

We also introduce a formalism for optimal sensor parameter selection for state estimation. The optimality is defined in terms of reduction in ambiguity or error in state estimation process. The main assumption is that state estimation becomes more reliable and accurate if the ambiguity/error in the underlying state estimation process can be minimized. We investigate this from an information theoretic perspective [1] where information about the context attribute is made available to the fusion center by a set of smart sensors. The fusion center produces an estimate of the state of the situation based on the intelligent analysis of the received data. We assume that the noisy observation across sensors are independent and identically distributed (i.i.d) random variable conditioned on the binary situation $\mathcal{R}$ (assume situation $\mathcal{R}$ here as binary for ease of modeling). Now each sensor attribute has a source entropy rate $H\left(a_{i}\right)$. Any sensor wishing to report this attribute $a_{i}$ must send $H\left(a_{i}\right)$ bits per unit time which is the entropy of the source being measured assuming that the sensor is sending the 'exact' physical state. Of course, different sensors (due to perhaps their sensing limitations) contribute in different measures to the 'error' in state estimation. So, the problem is to minimize the error (or keep it within a specified bound), while not exceeding the shared link rate capacity $\mathcal{Q}$. Thus by maximizing the posteriori detector probability we can minimize the estimation error of the random variables to accurately reconstruct the state of the situation.

Problem 1: Let $B$ be the vector of sensors and $A$ be the set of attributes, then imagine a $(B \times A)$ matrix where $B_{m i}=$ 1 where sensor $m$ sends attribute $a_{i}$. Then, the goal is to find a matrix $(B \times A)$ within the capacity constraint $\mathcal{Q}$ which minimizes the estimation error of the situation space.

$\sum_{m} \sum_{i} H\left(a_{i}\right) * B_{m i}<\mathcal{Q}$ and minimize $\left[P_{e}=P\{\tilde{\mathcal{R}} \neq \mathcal{R}\}\right]$

where $\tilde{\mathcal{R}}$ is an estimate of the original state $\mathcal{R}$

We also observe that the Chernoff information [1] at the fusion center is monotonically increasing in the number of sensors for a fixed decision rule $\pi$. State estimation error can be minimized by augmenting the number of sensors in $\pi$ until the capacity constraint $\mathcal{Q}$ is met with equality. The strategy $\pi$ being arbitrary, we conclude that having $\mathcal{Q}$ identical sensors in the $(B \times A)$ matrix, each sending one bit of information is optimal in terms of reducing the state estimation error. This configuration also conveys that the gain offered through multiple sensor fusion exceeds the benefits of getting detailed information from each individual sensor.

\section{REFERENCES}

[1] T. M. Cover and J. A. Thomas, "Elements of Information Theory", New York: Wiley, 1991

[2] A. Deshpande, C. Guestrin, S. Madden, "Using Probabilistic Models for Data Management in Acquisitional Environments", Proc. of the 2nd Biennial Conference on Innovative Data Systems Research (CIDR) pp. 317-328, Jan 2005.

[3] W. Hu, A. Misra and R. Shorey, "CAPS: Energy-Efficient Processing of Continuous Aggregate Queries in Sensor Networks", Fourth IEEE Int'l Conference on Pervasive Computing and Communications (PerCom), pp. 190-199, 2006.

[4] S. Padovitz, W. Loke, A. Zaslavsky, C. Bartolini and B. Burg, "An approach to Data Fusion for Context Awareness", 5th Int'l and Interdisciplinary Conference, CONTEXT 2005, pp. 353-367

[5] N. Roy, C. Julien and S. K. Das, "Resource-Optimized Ambiguous Context Mediation for Smart Healthcare", Technical Report TR-UTEDGE2008-011, UT-Austin, 2008.

[6] N. Roy, G. Pallapa and S. K. Das,"A Middleware Framework for Ambiguous Context Mediation in Smart Healthcare Application", Proc. of IEEE Int'l Conf. on Wireless and Mobile Computing, Networking and Communications (WiMob), Oct 2007. 